\title{
Case Report Ruptured Cerebral Aneurysm of Fenestrated A1 Segment of the Anterior Cerebral Artery: Case Report and Literature Review
}

Junji Uno, Ryosuke Otsuji, Nice Ren, Shintaro Nagaoka, Katsuharu Kameda, Kazushi Maeda, Yoshiaki Ikai, and Hidefuku Gi

Objective: Arterial fenestration is an unusual anatomic variation and is often associated with aneurysms. Aneurysm arising from the fenestration of the horizontal segment of the anterior cerebral artery (A1) is considered to be rare.

Case Presentation: We report a case of a 61 -year-old man who presented with a subarachnoid hemorrhage secondary to a ruptured aneurysm of fenestrated A1 segment of the anterior cerebral artery. Cerebral angiogram revealed an aneurysm at right $\mathrm{A} 1$ fenestration. The aneurysm was embolized by coils, leading to complete occlusion, while preserving both channels of the fenestration. On the 11th postoperative day, the patient suffered cerebral embolic stroke due to paroxysmal atrial fibrillation and his left proximal middle cerebral artery was occluded. He is doing rehabilitation for right hemiparesis and aphasia but remains severely disabled.

Conclusion: Considering that dissection of fenestrated A1 aneurysms is sometimes difficult, and there are several branches from one or both channels of fenestration, endovascular therapy would have some advantages over the neck clipping.

Keywords $>$ fenestration, anterior cerebral artery, cerebral aneurysm

\section{Introduction}

A fenestration is a division of the arterial lumen, resulting in separate channel, each with its own endothelial layer and muscularis tunica; the adventitial layer may or may not be shared between channels. ${ }^{1)}$ Cerebral aneurysms in the horizontal segment of the anterior cerebral artery (A1) are relatively rare $(0.88 \%){ }^{2)}$ Fenestrations accompanying cerebral aneurysms are well-known vascular malformations. However, there have been few reports on fenestrations in the A1 segment, and the etiology of A 1 fenestrations is less clear than that of basilar and vertebral artery fenestrations. ${ }^{1)}$

Department of Neurosurgery, Baba Memorial Hospital, Sakai, Osaka, Japan

Received: November 2, 2016; Accepted: January 13, 2017 Corresponding author: Junji Uno. Department of Neurosurgery, Baba Memorial Hospital, 4-244 Hamaderafunaochou-Higashi, Nishi, Sakai, Osaka 592-8341, Japan Email: babahpns@silver.ocn.ne.jp

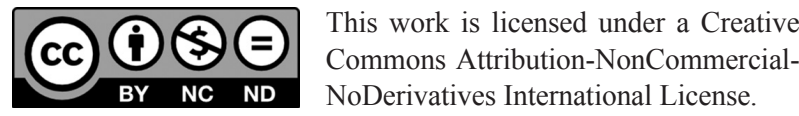

(C)2017 The Japanese Society for Neuroendovascular Therapy
Most of these aneurysms have been treated with clipping. Together with a discussion of the literature, we report a case of a ruptured cerebral aneurysm in the fenestrated segment of A1 treated with coil embolization.

\section{Case Presentation}

A 61-year-old male, a hypertensive smoker, presented with a sudden onset of disturbance of consciousness during sleep and was referred to our hospital. His medical history included smoking and spinal cord infarction treated with cilostazol. He had no history of atrial fibrillation. On admission, neurological examination was unremarkable except for meningeal signs and laboratory data demonstrated no abnormal findings. CT scan performed on the day of admission (day 0) revealed Fisher group 3 interhemispheric fissure-dominant diffuse subarachnoid hemorrhage (SAH) and a diagnosis of SAH of World Federation of Neurosurgical Societies grade I and Hunt and Kosnick grade II was made (Fig. 1). One day after admission (day 1), cerebral angiogram revealed a fenestration in the distal right A1 segment, and a cerebral aneurysm $3.5 \mathrm{~mm} \times$ $4.0 \mathrm{~mm}$ in size arising from the proximal end of the 


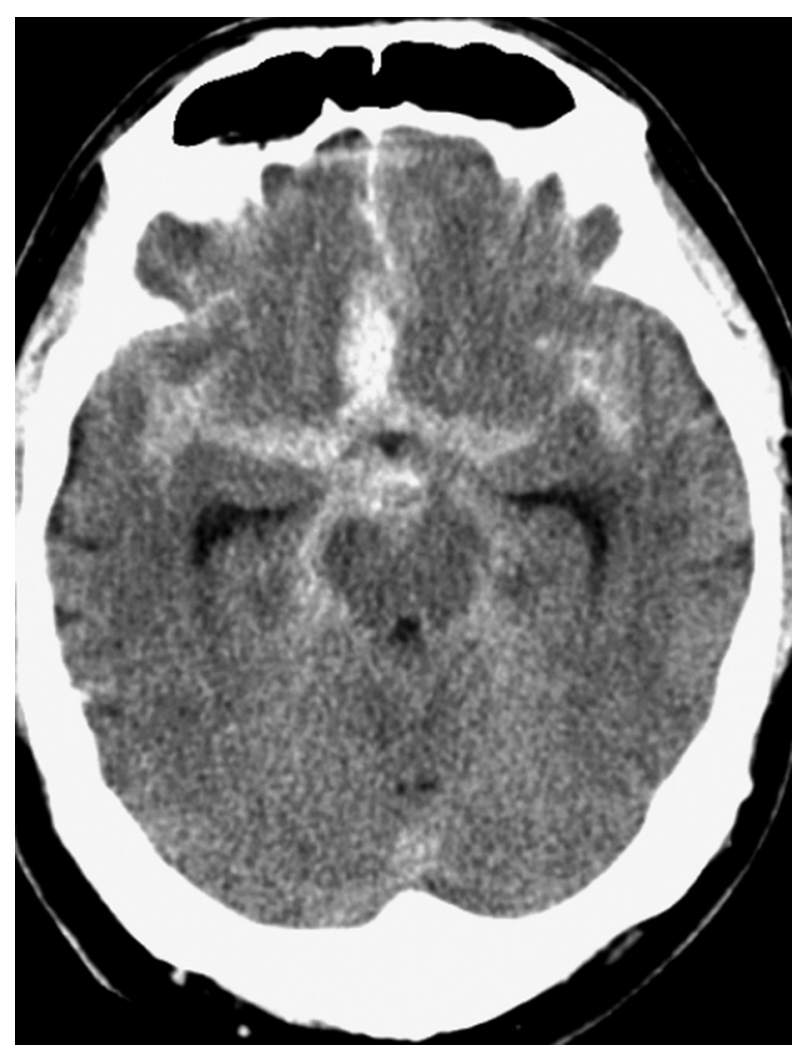

Fig. 1 Computed tomography on admission showing subarachnoid hemorrhage in the basal cistern and inter-hemispheric fissure.

fenestration (Figs. 2A and 2B). Recurrent artery of Heubner was found at A1-2 junction. No cerebral aneurysms were found at any other site. The left A1 segment was not hypoplastic. On day 2, endovascular embolization of the aneurysm was performed with the patient under general anesthesia. Using Orbit Galaxy Complex Extra Soft Detachable Coils $3.5 \mathrm{~mm} \times 7.5 \mathrm{~cm}$ (Codman \& Shurtleff, Raynham, MA, USA), ED coil-10 Extra Soft type R $2.5 \mathrm{~mm}$ $\times 3 \mathrm{~cm}$ (Kaneka Medix, Osaka, Japan), and Target Nano 360 Detachable Coils $1.5 \mathrm{~mm} \times 2 \mathrm{~cm}$ (Stryker Neurovascluar, Fremont, CA, USA), total occlusion of the aneurysm was achieved (Fig. 3). The volume embolization rate was $32 \%$. Visualization of the duplicated A1 segments forming the fenestration remained good.

\section{Postoperative course}

The patient awoke from anesthesia and did not exhibit any symptom of neurological deficit postoperatively. He progressed well but suddenly exhibited atrial fibrillation on his electrocardiogram (ECG) on postoperative day 11 (day 13). Right hemiparesis and aphasia subsequently appeared. Emergency cerebral angiography was performed, revealing occlusion of the proximal left middle cerebral artery.
Cerebral embolism due to atrial fibrillation was diagnosed, and mechanical thrombectomy was successfully performed to recanalize left middle cerebral artery. Left internal carotid angiogram indicated that the cerebral aneurysm had been embolized, and both fenestrated A1 and A2 channels were well visualized. A follow-up CT scan revealed extensive infarction in the territory of left middle cerebral artery. The patient's right hemiparesis and aphasia persisted, and he is currently undergoing rehabilitation.

\section{Discussion}

The most common sites for fenestrations of intracranial arteries to develop are, in decreasing order, the vertebral artery, basilar artery, and the middle cerebral artery. ${ }^{1)}$ San-Galli et al. ${ }^{3)}$ have reported that fenestrations are most common in the anterior communicating artery. There have been few reports on fenestrations occurring in the anterior cerebral artery. Fawcett et al. ${ }^{4)}$ reported a frequency of $0.14 \%$ in autopsy cases. Fenestrations are often accompanied by other cerebrovascular abnormalities. Suzuki et al. ${ }^{2)}$ reviewed 38 cases of A1 aneurysms and found an incidence of 15.8\% $(6 / 38)$ for A1 fenestration. Friedlander et al. ${ }^{5)}$ detected cerebral aneurysms in $16(27.6 \%)$ of 58 cases of A1 fenestrations. Meanwhile, Sanders et al. ${ }^{1)}$ reported that according to cerebral angiogram, aneurysms that develop in fenestrations exhibit the same association as those that develop in normal bifurcation sites. In most cases, aneurysms develop proximal edge of the fenestration, and a histological examination of the fenestration tissue has shown that the loss of tunica media at the proximal edge of the fenestration makes cerebral aneurysms prone to develop at that site. ${ }^{6-8)}$ Similarly, other histological examinations of fenestrations have indicated that there is tunica media loss not only at the proximal edge of the fenestration but also at the distal edge. ${ }^{89}$ ) In addition, flow phenomena at the proximal end of fenestrations, where hemodynamic stress and increased turbulence are present, may contribute to aneurysm formation. ${ }^{10)}$

The pathophysiologic mechanism for the development of A1 fenestration is not clearly explained. According to Padget's illustration of a $14-\mathrm{mm}$ embryo, ${ }^{11)}$ there is a plexiform anastomosis between the anterior cerebral artery (ACA) and the primitive olfactory artery. In 18- and 24-mm embryos, there is a fenestration of the distal end of the ACA which disappears by the $43-\mathrm{mm}$ stage. During the 18 - to 43-mm embryos, fenestration of the distal A1 segment might occur due to the lack of fusion of the plexiform anastomosis present in the distal primitive ACA. ${ }^{5)}$ Teal et al. ${ }^{12)}$ suggested 

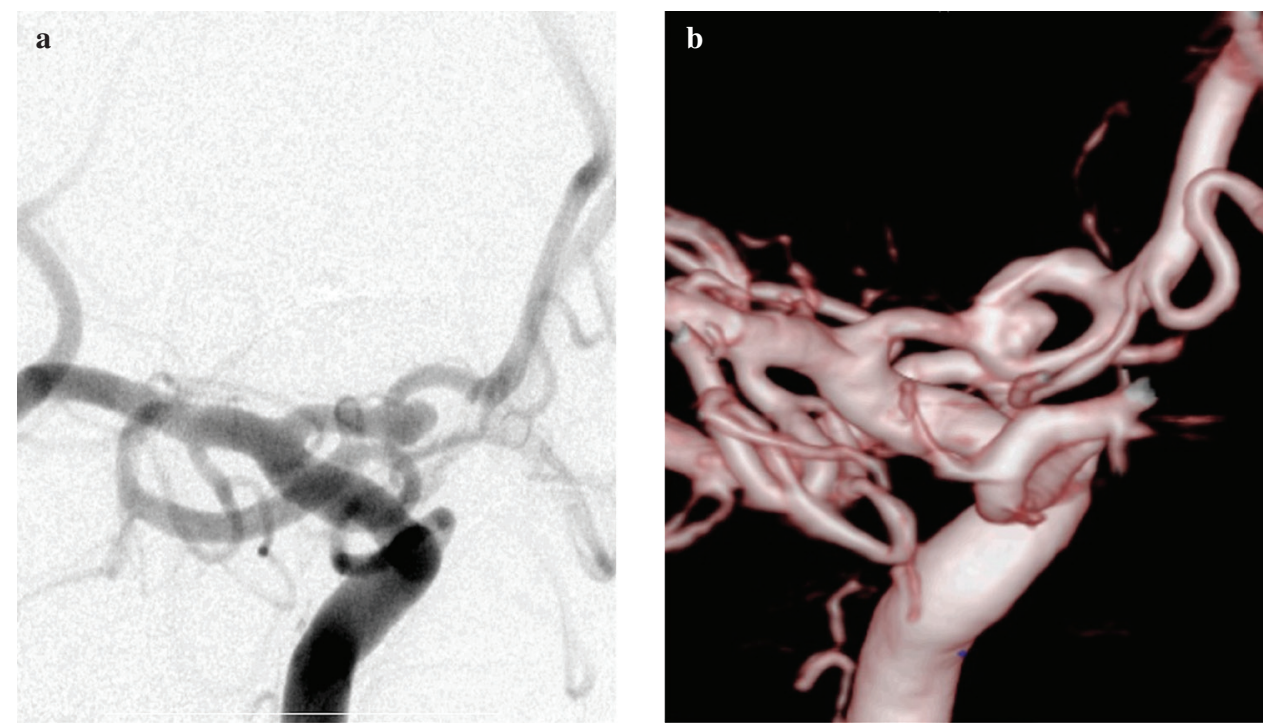

Fig. 2 (a) Preoperative right carotid angiogram showing fenestrated A1 segment of the anterior cerebral artery associated with cerebral aneurysm in an oblique plane. (b) The 3D DSA clearly illustrating a fenestration of $\mathrm{A} 1$ segment with the aneurysm located in the proximal end of it.

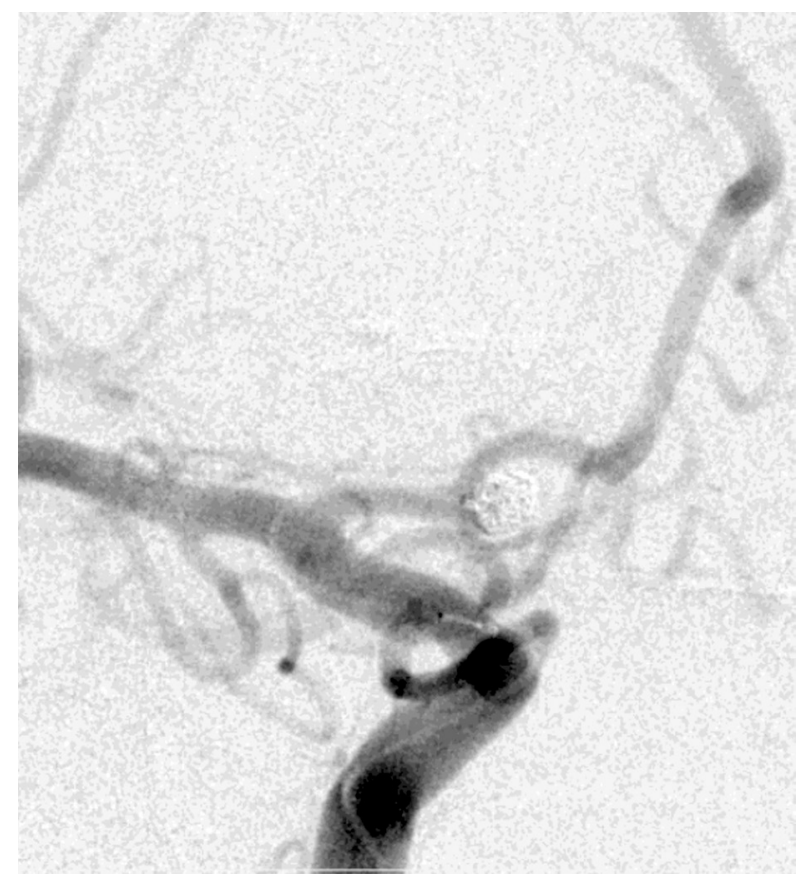

Fig. 3 Postoperative right carotid angiogram showing complete occlusion of aneurysm and preservation of both channels of the fenestration.

that fenestrations in internal carotid system may result from true partial duplication, incomplete fusion of the network of vessels which serves as the precursor of a solitary named vessels, or incorporation of a nerve within the definitive artery.

Including our patient, there have been 19 such cases reported in the past (Table 1). The mean age of the patients was 54.4 years, and the male-to-female ratio was $1: 1$. The right-to-left ratio was 12:7, and there were 16 cases of ruptured aneurysms. The mean age of the patients with ruptured aneurysms was 56 years, suggesting a tendency for onset in slightly younger patients than those of SAH in general. Kwon et al. ${ }^{13)}$ developed classifications based on the site of aneurysms. Type I refers to aneurysms on the proximal end of the fenestration; Type II refers to aneurysms arising from the duplicated A1 trunk, and Type III refers to multiple aneurysms within the fenestrated A1. In addition to these classifications, we suggested Type IV to refer to aneurysms at the distal end of the fenestration, with Koh et al. ${ }^{14)}$ reporting a case of the aneurysm at this site. Type I was the most common type, accounting for 14 of 19 cases. Type II only accounted for three cases, and an investigation of images available in the literature revealed that this type often involves cases with narrow space between channels of fenestration. Type III only accounted for one case with multiple aneurysms. Our patient was classified as Type I. Regarding treatment, many patients underwent clipping, which has been reported to offer stable treatment outcomes. However, it is reportedly difficult to perform whether there is strong adhesion between the aneurysm dome and fenestration vessel or when there is a perforating branch from the fenestration ${ }^{15)}$ as in the case of aneurysms of the proximal (M1) segment of the middle cerebral artery. An average 6.4 perforators arise from the A1 segment, and divide to yield average 21.9 vessels. ${ }^{16}$ ) The possibility of 


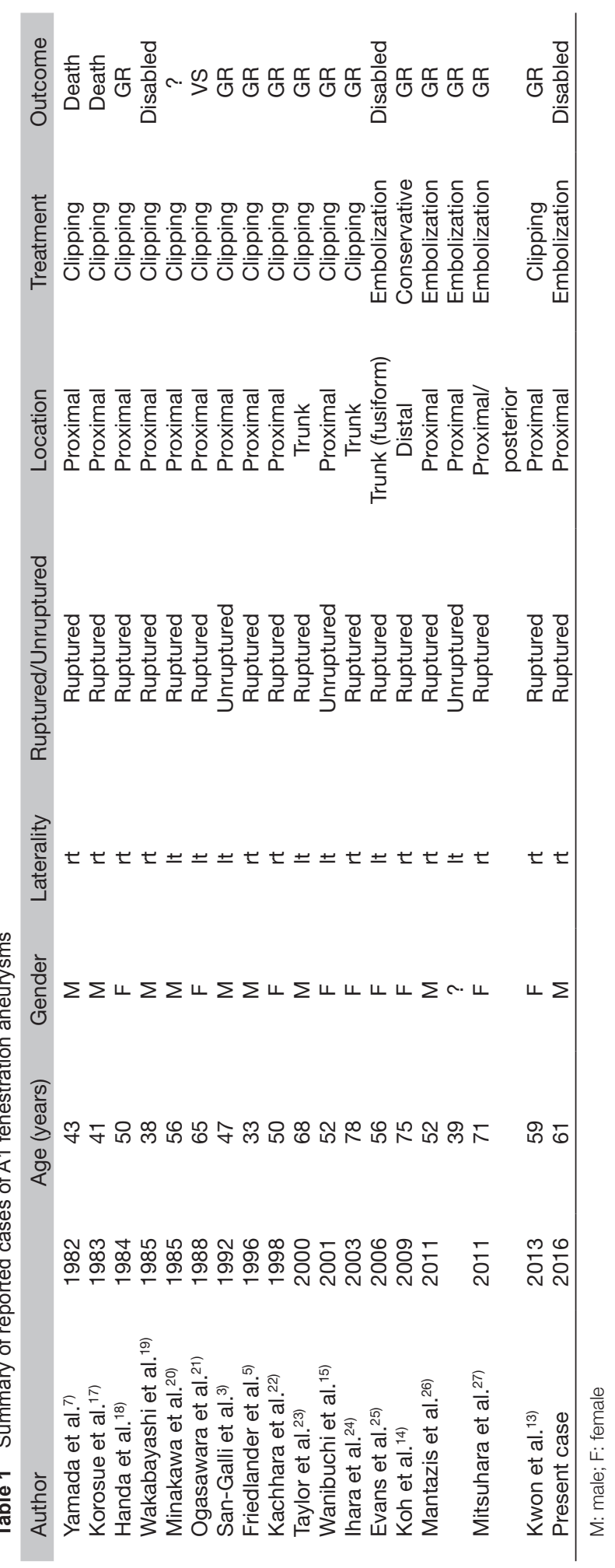


branches from channels of fenestration can be easily assumed. There have also been recent reports on coil embolization, which has resulted in favorable outcomes. Considering that in the case treated by neck clipping of the aneurysm, dissection of the fenestration aneurysms is sometimes challenging and the perforating branch is sometimes sacrificed, the benefits of coil embolization can be understood. It could be particularly useful for Type I cases in which the dome of aneurysm is adherent to surrounding fenestration channels. The embolization performed on our patient was not technically difficult, and the aneurysm was successfully embolized. The patient progressed well postoperatively. On postoperative day 11, paroxysmal atrial fibrillation appeared on his ECG, after which cerebral embolism occurred. However, as the embolism was of the opposing side middle cerebral artery, we do not believe that it was related to the treatment we implemented. The patient had no history of atrial fibrillation and did not undergo any anticoagulation therapy pre- or postoperatively.

Many cases of cerebral aneurysms in fenestration sites involve ruptured aneurysms, and were small size of aneurysms which are prone to rupture. ${ }^{15)}$ Aggressive treatment should be considered if the aneurysm is detected when unruptured. Furthermore, the fenestration site may not necessarily show up on performing cerebral angiography. If an aneurysm is noted in the A1 segment, a treatment policy should be considered, keeping the possibility of a fenestration in mind.

\section{Conclusion}

We treated a 61-year-old man who developed a ruptured aneurysm in the A1 fenestration. A1 fenestration is rare, and there have been few reports on cerebral aneurysms at this site. Although coil embolization was successful and the patient progressed well, he also suffered from cerebral embolism caused by paroxysmal atrial fibrillation leading to right hemiparesis and aphasia. As there have also been reports indicating that cerebral aneurysms that occur in fenestrations are likely to rupture, aggressive treatment should be considered if an unruptured aneurysm is detected.

\section{Disclosure Statement}

None of the authors or co-authors have any conflict of interest to declare.

\section{References}

1) Sanders WP, Sorek PA, Mehta BA: Fenestration of intracranial arteries with special attention to associated aneurysms and other anomalies. AJNR Am J Neuroradiol 1993; 14: 675-680.

2) Suzuki M, Onuma T, Sakurai Y, et al: Aneurysms arising from the proximal (A1) segment of the anterior cerebral artery. A study of 38 cases. J Neurosurg 1992; 76: 455-458.

3) San-Galli F, Leman C, Kien P, et al: Cerebral arterial fenestrations associated with intracranial saccular aneurysms. Neurosurgery 1992; 30: 279-283.

4) Fawcett E, Blachford JV: The circle of willis: An examination of 700 specimens. J Anat Physiol 1905; 40: 63.2-70.

5) Friedlander RM, Oglivy CS: Aneurysmal subarachnoid hemorrhage in a patient with bilateral A1 fenestrations associated with an azygos anterior cerebral artery. Case report and literature review. J Neurosurg 1996; 84: 681-684.

6) Crompton MR: The pathology of ruptured middlecerebral aneurysms with special reference to the differences between the sexes. Lancet 1962; 2: 421-425.

7) Yamada $T$, Inagawa $T$, Takeda $T$ : Ruptured aneurysm at the anterior cerebral artery fenestration. Case report. J Neurosurg 1982; 57: 826-828.

8) Black SP, Ansbacher LE: Saccular aneurysm associated with segmental duplication of the basilar artery. A morphological study. J Neurosurg 1984; 61: 1005-1008.

9) Finlay HM, Canham PB: The layered fabric of cerebral artery fenestrations. Stroke 1994; 25: 1799-1806.

10) Hacein-Bey L, Muszynski CA, Varelas PN: Saccular aneurysm associated with posterior cerebral artery fenestration manifesting as a subarachnoid hemorrhage in a child. AJNR Am J Neuroradiol 2002; 23: 1291-1294.

11) Padget DH: The development of the cranial arteries in the human embryo. Contrib Embryol 1948; 32: 205-262.

12) Teal JS, Rumbaugh CL, Bergeron RT, et al: Angiographic demonstration of fenestrations of the intradural intracranial arteries. Radiology 1973; 106: 123-126.

13) Kwon WK, Park KJ, Park DH, et al: Ruptured saccular aneurysm arising from fenestrated proximal anterior cerebral artery : case report and literature review. J Korean Neurosurg Soc 2013; 53: 293-296.

14) Koh JS, Kim EJ, Lee SH, et al: Ruptured aneurysm arising from the distal end of a proximal a (1) fenestration : case report and review of the literature. J Korean Neurosurg Soc 2009; 45: 43-45.

15) Wanibuchi M, Kurokawa Y, Ishiguro M, et al: Characteristics of aneurysms arising from the horizontal portion of the anterior cerebral artery. Surg Neurol 2001; 55: 148-154; discussion 154-155.

16) Rosner SS, Rhoton AL, Ono M, et al: Microsurgical anatomy of the anterior perforating arteries. $J$ Neurosurg 1984; 61: 468-485. 
17) Korosue K, Kuwamura K, Okuda Y, et al: Saccular aneurysm arising from a fenestrated anterior cerebral artery. Surg Neurol 1983; 19: 273-275.

18) Handa J, Nakasu $Y$, Matsuda $M$, et al: Aneurysms of the proximal anterior cerebral artery. Surg Neurol 1984; 22: 486-490.

19) Wakabayashi T, Tamaki N, Yamashita H, et al: Angiographic classification of aneurysms of the horizontal segment of the anterior cerebral artery. Surg Neurol 1985; 24: 31-34.

20) Minakawa $T$, Kawamata $M$, Hayano $M$, et al: Aneurysms associated with fenestrated anterior cerebral arteries. Report of four cases and review of the literature. Surg Neurol 1985; 24: 284-288.

21) Ogasawara $H$, Inagawa $T$, Yamamoto $M$, et al: Aneurysm in a fenestrated anterior cerebral artery-case report. Neurol Med Chir (Tokyo) 1988; 28: 575-578.

22) Kachhara R, Nair S, Gupta AK: Fenestration of the proximal anterior cerebral artery (A1) with aneurysm manifesting as subarachnoid hemorrhage-case report. Neurol Med Chir (Tokyo) 1998; 38: 409-412.
23) Taylor R, Connolly ES, Duong H: Radiographic evidence and surgical confirmation of a saccular aneurysm on a hypoplastic duplicated A1 segment of the anterior cerebral artery: case report. Neurosurgery 2000; 46: 482-484.

24) Ihara S, Uemura K, Tsukada A, et al: Aneurysm and fenestration of the azygos anterior cerebral artery-case report. Neurol Med Chir (Tokyo) 2003; 43: 246-249.

25) Evans AL, Corkill RA, Wenderoth JD: Ruptured fusiform aneurysm of fenestrated A1 segment of the anterior cerebral artery. Case report and review of the literature. Neuroradiology 2006; 48: 196-199.

26) Mantatzis M, Kizilkilic O, Albayram S, et al: Endovascular treatment of aneurysms associated with fenestrated A1 segment of anterior cerebral artery: report of two cases. $\mathrm{J} \mathrm{Neu-}$ roimaging 2011; 21: 165-169.

27) Mitsuhara T, Sakamoto S, Kiura Y, et al: Endovascular coil embolization for ruptured kissing aneurysms associated with A1 fenestration. Surg Neurol Int 2011; 2: 85. 